ÉGYPTE

monde arabe

\section{Égypte/Monde arabe}

9 | 1992

Vers une économie libérale?

\title{
Investissements étrangers au Maghreb
}

Les risques d'un désengagement durable

\section{Larbi Talha}

\section{(2) OpenEdition}

\section{Journals}

Édition électronique

URL : https://journals.openedition.org/ema/1216

DOI : 10.4000/ema.1216

ISSN : 2090-7273

Éditeur

CEDEJ - Centre d'études et de documentation économiques juridiques et sociales

Édition imprimée

Date de publication : 31 mars 1992

Pagination : 67-82

ISSN : 1110-5097

Référence électronique

Larbi Talha, «Investissements étrangers au Maghreb », Égypte/Monde arabe [En ligne], 9 | 1992, mis en ligne le 08 juillet 2008, consulté le 07 juillet 2022. URL : http://journals.openedition.org/ema/1216 ; DOI : https://doi.org/10.4000/ema.1216

Ce document a été généré automatiquement le 7 juillet 2022

Tous droits réservés 


\title{
Investissements étrangers au Maghreb
}

\author{
Les risques d'un désengagement durable
}

\section{Larbi Talha}

1 Les temps ont changé : la méfiance ombrageuse affichée à l'égard du capital étranger par les pays maghrébins au lendemain de leur indépendance, a laissé place aujourd'hui à une stratégie d'ouverture tous azimuts et de libéralisme débridé. Tout est fait pour attirer les capitaux extérieurs et la législation a été modifiée en ce sens, comme en témoignent notamment les nouveaux codes d'investissements et les lois diverses promulguées durant les années 80 .

2 Au Maroc, de nouvelles mesures pour attirer le capital étranger sont prises dès 1983, avec la promulgation d'une nouvelle loi sur les investissements dans l'industrie : celleci n'exige plus que $50 \%$ du capital d'une société soient détenus par les nationaux et la garantie du transfert du capital et des dividendes est partiellement étendue aux résidents étrangers. Tout au long de la décennie 80, d'autres mesures viendront s'ajouter, visant notamment à accorder de nouveaux avantages fiscaux et financiers (allégements, prêts sans intérêts, etc..) pour réduire le coût de l'investissement et l'orienter vers des secteurs jugés prioritaires, tel que le tourisme par exemple.

3 En Tunisie, l'ensemble des codes d'investissements ont été profondément remaniés, à partir du milieu des années 80 , dans le sens de :

- la suppression des agréments préalables de la part des autorités et la simplification des formalités ;

- l'introduction d'avantages fiscaux très importants, en faveur notamment des investissements dans les secteurs exportateurs;

- la suppression de la règle des $50 \%$ dans tous les secteurs, sauf la production agricole ;

- la liberté totale de transfert du capital et des bénéfices et une grande liberté de change.

En Algérie, les mesures de libéralisation n'ont véritablement été entamées qu'à la fin des années 80, avec la loi de 1988, autorisant les entreprises publiques économiques, anciennes sociétés d'État, à contracter avec des partenaires étrangers pour des joint- 
venture. Les limites à la participation étrangère dans les sociétés mixtes sont supprimées fin 1989.

5 En avril 1990, la Loi sur la Monnaie et le Crédit, véritable révolution, parachève le dispositif d'ouverture totale aux capitaux étrangers :

- en autorisant les non-résidents à importer des capitaux, soit pour des investissements directs, soit pour des investissements de portefeuille, et à rapatrier le principal et les bénéfices générés par ces investissements.

- en supprimant l'obligation de la participation de $51 \%$ du secteur public algérien ;

- en autorisant les banques étrangères à s'implanter dans le pays.

Une loi rectificative de Finances votée en 1990 ouvre également les activités de commerce aux capitaux étrangers. Et enfin, une nouvelle loi sur les hydrocarbures votée le 30 novembre 1991 autorise les sociétés étrangères à participer jusqu'à hauteur de $49 \%$ à l'exploitation des gisements déjà en production ou à découvrir. Dans le cas de ce dernier pays, l'Algérie, il s'agit là plus que d'un simple changement de politique à l'égard du capital étranger, c'est une véritable révolution sinon dans les faits, du moins dans les mentalités.

7 Les facteurs qui ont contraint les trois pays à ce retournement sont connus : il y a d'abord le poids étouffant de la dette extérieure, associé à une crise aiguë du financement interne due à l'effondrement des recettes extérieures, il y a ensuite l'assèchement de l'offre de crédit international dû au choc monétariste du début des années 80 et à la raréfaction de l'offre de capitaux sur des marchés financiers fortement déréglés et, last but not least, il y a l'entrée en lice d'une nouvelle périphérie en voie de constitution à partir de l'éclatement de l'ancien "bloc des pays de l'Est», et puissamment concurrentielle de l'ancienne périphérie des pays du Sud.

8 Cet infitah maghrébin sera-t-il plus attractif pour les capitaux étrangers que son antécédent égyptien? Il est trop tôt pour répondre à cette question. Aussi, le but de cet article sera, non pas d'évaluer les premiers résultats enregistrés en matière d'investissements extérieurs, mais de voir quelles leçons tirer de leur évolution passée, compte tenu de :

1. L'importance de leur montant et de leur origine géographique.

2. Leur répartition par pays et par secteur d'activité.

3. Leurs effets induits sur les configurations du régime d'accumulation dans chaque pays.

\section{Flux de capitaux et investissements directs au Maghreb : bref aperçu}

9 Quelle est la place des investissements directs dans l'ensemble des flux de capitaux ? Un rapide coup d'oeil sur la balance des capitaux nous permettra d'introduire quelques éléments d'appréciation préalablement à toute analyse.

En dépit de la difficulté à réunir des séries longues et homogènes sur cette question, les chiffres rassemblés dans les tableaux 1,2 et 3 , pour incomplets qu'ils soient, permettent de tirer trois constatations. 


\section{$1^{\text {ère }}$ constatation : excepté la Libye, les pays du Maghreb sont globalement importateurs nets de capitaux}

11 C'est du moins ce qu'on peut observer à la lecture du tableau 1 ci-après : les chiffres indiquent ici le total des investissements directs et de portefeuille, les emprunts de capitaux à long et court terme, ainsi que les placements bancaires à court terme. Le solde est positif pour les années retenues et pour les quatre pays, sauf pour la Libye en 1980. La chute observée durant les années 80 (Algérie:-67\%, Maroc:-83\% et Tunisie :- $52 \%$ ), venant après le mouvement de hausse des années 70 , n'est pas pour surprendre, si on replace le phénomène dans le contexte des tendances mondiales évoquées plus haut.

1-Balance des capitaux en millions \$

\begin{tabular}{|l|r|r|r|}
\hline & 1970 & 1980 & 1987 \\
\hline ALGERIE & 68 & 955 & 310 \\
LIBYE & 923 & -865 & 150 \\
MAROC & 140 & 1156 & 188 \\
TUNISIE & 72 & 373 & 177 \\
\hline
\end{tabular}

Source : Statistiques financières internationales - Yearbook -1970 à 1989 directs à l'étranger (en 1980, par exemple, le montant de ceux-ci était de 1162 millions), de prêts à court terme (107 millions \$ en 1985). En 1987, cependant, ce pays a lui aussi fait appel aux ressources extérieures : 290 millions $\$$ ont été empruntés à court terme sur les places financières.

14 En Algérie, la balance des capitaux a enregistré une sortie nette de 121 millions \$ en 1985 , correspondant probablement aux remboursements de la dette : 34 millions $\$$ au titre de la dette à court terme et 85 millions $\$$ pour la dette à long terme. En revanche, en 1987, ce pays a été obligé d'emprunter à nouveau pour un montant de 289 millions \$ pour faire face au service de la dette. Rappelons à cet égard que la dette algérienne, au contraire de celle de la Tunisie et du Maroc, est à $80 \%$ d'origine privée et que l'échéance moyenne s'alourdit considérablement au fil des années, passant de 9,5 ans à 3,5 ans ${ }^{1}$ en fin 1989.

\section{$2^{\mathrm{e}}$ constatation : la majeure partie des capitaux est fournie par l'Europe, mais leur montant n'a jamais été très important}

Fonds publics et fonds privés réunis, les apports nets cumulés sur la période 1976-1987 sont à $70 \%$ d'origine européenne en ce qui concerne l'Algérie, $56 \%$ en ce qui concerne la Tunisie et $45 \%$ en ce qui concerne le Maroc, ce dernier pays bénéficiant d'une aide plus substantielle de la part des pays du Golfe. La part de l'Europe est encore plus écrasante lorsqu'on distingue secteur public et secteur privé: les 3/4 des flux nets d'origine publique sont européens et les apports nets des capitaux privés européens

Égypte/Monde arabe, 9 | 1992 
représentent $85 \%$ du total des ressources privées dans le cas du Maroc et $98 \%$ dans le cas de la Tunisie.

2-Flux nets cumulés 1976-1987 en millions \$

\begin{tabular}{|c|r|r|r|}
\hline & Algérie & Maroc & Tunisie \\
\cline { 2 - 4 } Apports nets du secteur public (tolal) & 4020 & 9617 & 3723 \\
Pays du CAD & 3144 & 4161 & 2088 \\
dont la France & 681 & 2136 & 484 \\
Pays de rOPEP & -141 & 2685 & 361 \\
(Pays de la CEE) & $(1510)$ & $(3221)$ & $(1634)$ \\
Apports neis du secteur privé des pays & & & \\
du CAD (lotal) & 8897 & 2705 & 1135 \\
dont la France & 3429 & 1740 & 867 \\
dont les autres CEE & 4327 & 558 & 236 \\
\hline
\end{tabular}

- Comilé d’aide au développement. Source: voir note (2)

Il faut souligner ici que les ressources privées constituent principalement des prêts bancaires accordés en soutien des exportations (prêts liés), car, comme l'expliquent A. Chevallier et V. Kessler, les investissements directs des entreprises à proprement parler représentent une part fort modeste, pour ne pas dire négligeable: «Au Maroc et en Algérie, ce type d'apports n'a pas dépassé respectivement $1 \%$ et $0,9 \%$ des recettes courantes. La contribution française des dix dernières années a été particulièrement faible, marquant un désintérêt manifeste pour le Maghreb: les trois pays réunis n'auraient reçu entre 1978 et 1987, que 74 millions de francs sur les 36 milliards d'investissements directs nets français dans les pays en développement $»^{2}$.

17 Ce désintérêt des investisseurs privés pour les pays maghrébins, qui, comme on le voit, n'est pas seulement d'ordre conjoncturel, s'est encore renforcé depuis que les pays de l'ex-bloc de l'Est sont en passe de devenir une formidable zone d'attraction pour les capitaux européens entraînant un effet d'éviction de plus en plus redouté par les pays du Sud. Un rapport patronal (le rapport Prouteau) réalisé en 1991 constate cette désaffection et s'inquiète de la difficulté de relancer les investissements privés, au Maroc et en Tunisie notamment.

18 Pourtant, c'est dans les pays du Maghreb que les investisseurs français réalisent leur meilleur score de rentabilité : une récente étude de la Banque de France montre en effet que le rendement total (dividendes encaissés + bénéfices réinvestis) des capitaux propres investis par les entreprises françaises dans le monde, a enregistré son taux le plus élevé au Maghreb (chiffres 1989) : 


\begin{tabular}{lr} 
Maghreb & $13,3 \%$ \\
Amérique Latine & $9,6 \%$ \\
CEE & $8,9 \%$ \\
dont Italie & $12,7 \%$ \\
\multicolumn{1}{c}{ Espagne } & $11,4 \%$ \\
\multicolumn{1}{c}{ RFA } & $7,8 \%$ \\
Pays de la zone Franc & $-1,8 \%$ \\
Amérique du Nord & $-2,7 \%$ \\
\multicolumn{1}{c}{ dont USA } & $-3,3 \%$
\end{tabular}

Source : cité par la Tribune de l'expansion 13/9/91, p. 11.

Soulignons que le ratio maghrébin est supérieur de 4,5 points au rendement moyen réalisé dans l'hexagone ( $13,3 \%$ contre $8,9 \%)$, et de 5,5 points à celui réalisé en Allemagne (7,8\%). Enfin, aux États-Unis les investisseurs français ont perdu de l'argent en 1989 : près de 3 milliards de francs, soit 3,3\% de leur encours. L'année 1989 auraitelle été exceptionnelle en matière de résultats financiers ? Il est difficile de répondre à la question, faute d'éléments de comparaison pour les autres années. On ne peut cependant s'empêcher d'observer que le critère du profit n'exerce pas, dans le cas du Maghreb, son pouvoir classique d'attraction. Pourquoi ? Serait-ce alors le risque que présenteraient ces pays qui serait à l'origine de la désaffection des investissements à leur égard ? L'Algérie, le Maroc et la Tunisie sont-ils des pays très « risqués »? 
3-Risque-pays établi en août 1991

\begin{tabular}{|l|l|c|c|c|c|}
\hline \multicolumn{2}{|c|}{ Pays/Risque } & Politique & Economique & Financier & Total $^{*}$ \\
\hline 1 & Suisse & 93,0 & 50,0 & 39,5 & 91,5 \\
49 & Arable saoudite & 60,0 & 31,0 & 35,5 & 63,5 \\
50 & Israēl & 58,0 & 33,0 & 34,5 & 63,0 \\
56 & Pologne & 62,0 & 29,0 & 31,0 & 61,0 \\
62 & Algérie & 54,0 & 30,0 & 32,5 & 58,5 \\
66 & Egypte & 54,0 & 30,0 & 29,0 & 56,5 \\
66 & Libye & 52,0 & 27,0 & 34,0 & 56,5 \\
70 & Syrie & 53,0 & 23,0 & 36,0 & 56,0 \\
71 & Iran & 56,0 & 28,0 & 26,5 & 55,5 \\
71 & Maroc & 52,0 & 28,0 & 30,5 & 55,5 \\
71 & URSS & 53,0 & 36,0 & 21,5 & 55,5 \\
75 & Tunisie & 54,0 & 23,0 & 32,0 & 54,5 \\
93 & Turquie & 52,0 & 19,0 & 27,5 & 49,5 \\
97 & Jordanie & 45,0 & 20,0 & 30,5 & 48,0 \\
104 & Koweit & 38,0 & 24,0 & 29,5 & 46,0 \\
118 & Liban & 32,0 & 11,0 & 35,0 & 39,0 \\
126 & Irak & 19,0 & 4,0 & 25,5 & 24,5 \\
127 & Soudan & 15,0 & 10,0 & 22,5 & 24,0 \\
\hline
\end{tabular}

- Le meilleur score correspondant a un risque nul est de 100 pour le risque poiltique et le risque total, et de 50 pour le risque économique el financier.

Source: The Wall Street Journal cilé par le Courrier intemational 24/10/91, p. 8.

20 Ce classement élaboré en août 1991 pour 129 pays par la branche américaine du International Business Communications de Londres et publié par l'International Country Risk Guide montre que la position des trois pays du Maghreb sur l'échelle mondiale du « risque-pays» est somme toute assez honorable : sur 129 pays retenus, l'Algérie est classée en $62^{\mathrm{e}}$ position, l'Égypte en $66^{\mathrm{e}}$ position à égalité avec la Libye, le Maroc vient à la $71^{\mathrm{e}}$ place et la Tunisie à la $75^{\mathrm{e}}$ place. Quant au Koweït et à l'Irak, ils sont classés respectivement à la $104^{\mathrm{e}}$ et à la $126^{\mathrm{e}}$ position. L'Arabie Saoudite, elle, est évidemment mieux placée, en $49^{\mathrm{e}}$ position.

\section{$3^{\mathrm{e}}$ constatation : le capital italien renforce sa présence au Maghreb, alors que le capital français se désengage}

21 Les calculs regroupés dans le tableau 4 (voir p. suivante) révèlent en effet ce double mouvement. Les investissements directs en provenance d'Italie augmentent régulièrement depuis 1980 dans les pays du Maghreb, avec une préférence très nette pour les marchés libyen et tunisien. Mais le Maroc et l'Algérie ont obtenu tout récemment des crédits importants de la part de ce pays, l'Italie, qui devient ainsi l'un des plus redoutables concurrents de la France. Celle-ci, on l'a vu, semble au contraire se désengager, nombre d'entreprises (400 filiales de 80 groupes français sont installées au Maghreb) liquidant leurs actifs dans ces pays. 


\section{Importance des investissements étrangers selon le pays et le secteur d'activité}

La présence du capital étranger est plus ancienne au Maroc, où la législation en ce domaine est restée plus libérale et les avantages offerts plus importants qu'ailleurs après l'Indépendance, qu'en Tunisie, où les investissements n'ont pris de l'ampleur qu'à partir des années 70 . En Algérie, en revanche, l'implantation du capital étranger, après les nationalisations des intérêts français, a été strictement limitée aux sociétés mixtes en association avec le secteur d'État, spécialement dans le secteur des hydrocarbures, et ce, jusqu'au début des années 80 .

La politique d'ouverture initiale par la Loi sur la Monnaie et le Crédit d'avril 1990 place désormais l'Algérie dans la course aux investissements étrangers, en concurrence directe avec le Maroc et la Tunisie, et ce, dans un contexte international où l'offre de capital est devenue rare et celle du travail abondante.

4-Investissements directs de la CEE dans WMA. En millions \$

\begin{tabular}{|c|c|c|c|c|c|c|c|c|c|c|c|c|}
\hline \multirow[b]{3}{*}{ Algérie } & \multicolumn{3}{|c|}{1970} & \multicolumn{3}{|c|}{1980} & \multicolumn{3}{|c|}{1985} & \multicolumn{3}{|c|}{1987} \\
\hline & Fce ${ }^{*}$ & Itadie & AFA & $F c \theta$ & ltalie & RFA & $F c \theta$ & Italie & RFA & $F \propto \infty$ & Itralie & AFA \\
\hline & $-74,5$ & & 0 & $-24,6$ & 5,1 & -297.1 & $-3,5$ & 3,3 & 97.1 & $-1,6$ & 4,4 & 86,2 \\
\hline Libye & 9.5 & & $-1,7$ & 4,0 & 255.8 & $-30,2$ & $-1,9$ & 383,4 & $-30,2$ & $-2,0$ & 463,2 & .40 .6 \\
\hline Maroc & $-2,5$ & & $-25,8$ & $-34,8$ & $3 t, 1$ & 37,9 & $.9,4$ & 43,8 & $.50,6$ & $.15,8$ & 67.1 & $-26,7$ \\
\hline Tunisie & $-2,1$ & & $-8,6$ & $-1 ;, 6$ & 36.9 & 21,4 & $\cdot 3,8$ & 40,1 & $-20,0$ & $-12,8$ & 101,4 & 9.4 \\
\hline Total UMA & -88.7 & & $-35,8$ & $-66,9$ & 329,0 & $-270,1$ & -18.7 & 470,7 & $-4,7$ & $-32,3$ & 636,1 & 27,2 \\
\hline
\end{tabular}

24 1. Au Maroc, le capital étranger, en dépit ou à cause de son implantation plus ancienne, ne représente, en fait, en termes de flux d'investissements, qu'une part modeste du total des investissements réalisés dans le pays. Celle-ci n'atteint pas en effet $4 \%$ au cours de la décennie 70 ( $2,4 \%$ en 1973 et 3,7\% en 1980). Les avantages offerts par le Code des Investissements de mars 1973 sont pourtant très alléchants ${ }^{3}$.

25 La tendance n'a pu jusqu'ici être redressée en dépit de la promulgation de différents Codes sectoriels en 1983, 1984 et en 1987, élargissant les garanties accordées au capital étranger. La part de ce dernier dans l'investissement global régresse même : de 3,7 \% en 1980, elle tombe à $2,6 \%$ en 1985 et à $3 \%$ en 1986. Tel est le premier constat. Faut-il en déduire que le rôle des investissements extérieurs dans l'économie du Maroc est d'une importance négligeable?

En fait, il faudrait aussi tenir compte de leur importance dans les secteurs d'activité qu'il contrôle. De ce point de vue, on peut faire une seconde observation : sous l'effet de la crise économique, le capital étranger délaisse les activités manufacturières devenues peu rentables et se concentre davantage dans les activités du tertiaire à rentabilité courte : transactions immobilières, activités du tourisme, intermédiation financière. 
Durant les années 70, les investissements étrangers représentaient près du tiers des investissements industriels au Maroc : 30 \% jusqu'en 1974, 35 \% en 1980. La décennie 80 a vu leur part chuter de moitié : celle-ci n'était plus en effet que de $17 \%$ en 1987 et de $15,4 \%$ en 1988. Le secteur du tourisme, en revanche, attire une proportion croissante du capital étranger : en dix ans, celle-ci a été multipliée par six, passant de $6 \%$ en $1972 / 73$ à $36,7 \%$ en $1982^{4}$. Ce mouvement n'a pu que se renforcer depuis cette date, car la privatisation des chaînes hôtelières à capitaux publics entamée depuis l'année 1985 constitue une aubaine pour des capitaux français ou moyen-orientaux à la recherche de profits vite réalisés.

En concurrence avec le secteur touristique, l'immobilier a connu un afflux d'investissements encore plus important : entre le quart et le tiers dés investissements étrangers réalisés durant la période $82 / 87$. D'un point de vue général, on peut observer que ce mouvement de mobilité intersectorielle du capital étranger semble obéir à un choix beaucoup plus affirmé pour les branches d'activités à faible intensité du capital, à bas salaires (le coût horaire au Maroc est estimé à 1,1 \$ contre 2,7 \$ en Tunisie par exemple) et tournées vers la demande extérieure.

L'examen de l'origine géographique des investissements extérieurs ne révèle pas de changements importants: quoiqu'en baisse relative, le capital français demeure prépondérant en dépit de la montée en puissance de l'apport saoudien et koweïtien depuis le premier choc pétrolier. Les investissements directs français représentent en effet $33 \%$ du total des investissements étrangers en 1970, $42 \%$ en 1974, 20,2 \% en 1982 et $16 \%$ en 1986. Dans le secteur manufacturier, le capital français représente $39 \%$ du capital étranger, et $71 \%$ des investissements originaires de la CEE.

La part des capitaux en provenance du Koweit et d'Arabie Séoudite n'a cessé d'augmenter depuis le premier choc pétrolier en 1974: au milieu des années 80, elle atteint autour de $17 \%$ du total des investissements étrangers au Maroc. Leur présence est fortement concentrée dans les activités du tourisme et de l'immobilier.

2. En Tunisie, la structure et l'importance des investissements étrangers ont connu un autre développement.

D’abord très méfiante à l'égard du capital étranger, la Tunisie a changé totalement de politique après la chute du gouvernement Ben Salah et l'échec de l'expérience du socialisme étatique des années 60. Deux lois notamment, promulguées l'une en 1972 et l'autre en 1974, consacrent l'ouverture aux investissements extérieurs. Des mesures très avantageuses favorisent alors une relance des flux qui allait prendre une ampleur remarquable durant la décennie 70 , puisque leur part dans l'investissement global atteint plus de $10 \%$ en moyenne durant cette période et monte jusqu'à $16 \%$ au début des années 80. Mais ici comme ailleurs, l'élan se trouve brisé par la crise économique qui affecte les économies maghrébines au début de la décennie 80 : en 1986, la part des investissements étrangers tombe à $9 \%$ (cf. tableau 5 ci-après). Différentes lois ont alors été promulguées durant les années 85-88 pour tenter d'encourager une nouvelle relance des investissements dans les secteurs notamment de l'énergie, des industries manufacturières, du tourisme et même de l'agriculture et de la pêche, mais semble-t-il en vain : 
5-Part du capital étranger dans l'investissement total et sectoriel en \%

\begin{tabular}{|l|r|r|r|r|r|}
\hline & 1973 & 1976 & 1980 & 1982 & 1986 \\
\cline { 2 - 6 } & 10,9 & 9,5 & 10,2 & 16,1 & 9,1 \\
Ensemble de léconomie & 0 & 0 & 1,8 & 1,8 & 3,2 \\
Tourisme et immobilier & 2,5 & 5,8 & 8,4 & 1,7 & 9,1 \\
Industrie manufacturière & 99,1 & 32,9 & 8,6 & 78,7 & 33,2 \\
Energie & & &
\end{tabular}

Source : d'après A. Chelbi et F. Charfi (1989) cités par Abdoun (R.) op. cit.

Les chiffres regroupés dans le tableau 5 montrent que, du point de vue de leur répartition sectorielle, la structure des investissements étrangers en Tunisie contraste nettement avec celle observée au Maroc: une très forte concentration dans le secteur de l'énergie, spécialement dans les activités pétrolières à forte intensité capitalistique, une présence négligeable dans les activités touristiques et les transactions immobilières et, ce, au moins jusqu'au début des années 80 , et enfin une intervention importante dans les industries manufacturières, principalement dans le textile où 11 à $15 \%$ du capital étranger est investi (en 1980 et 1986).

Au total, on constate que l'impact sectoriel est peu diversifié et ce, aussi bien en Tunisie qu'au Maroc. A quoi il faut ajouter que, dans l'un comme dans l'autre pays, le capital étranger a une préférence très nette pour les activités tournées vers l'exportation et le marché extérieur.

5 3. En Algérie, le capital étranger est également présent dans l'activité économique, mais cette présence revêt des formes tout à fait différentes. Si l'intervention des investisseurs privés étrangers a été pendant longtemps exclue, l'Algérie a, par contre, fait largement appel aux firmes transnationales pour la réalisation des projets industriels dans le cadre de sociétés mixtes. De la fin des années 60 au début des années 80 , une vingtaine environ de sociétés mixtes ont ainsi été créées, la plupart dans le secteur des hydrocarbures.

Mais d'autres formes d'interventions ont aussi été sollicitées. Au début de la décennie 70, les Sociétés nationales nouvellement créées ont fait appel aux firmes étrangères pour la fourniture de technologie (licences, brevets), pour des contrats d'assistance technique ou la vente d'équipements industriels. Durant la seconde phase de l'industrialisation (1974-1980), les multinationales ont également été sollicitées pour participer à la réalisation d'usines entières « clés en mains " puis " produits en mains », dans le cadre de contrats de vente globalisés.

7 Dans ce type de contrats, toute prise de participation étrangère est exclue alors que dans la formule des sociétés mixtes, elle existe mais sous forme minoritaire. L’Algérie pouvait alors se passer du concours direct des investissements extérieurs, les recettes tirées du pétrole lui permettant de financer, avec l'aide des prêts levés sur le marché international, ses projets d'industrialisation et, ce, jusqu'au deuxième contre-choc pétrolier qui allait précipiter l'Algérie dans la crise.

8 La chute brutale de la rente pétrolière en 1986, qui vient s'ajouter à une stagnation durable de la production pétrolière, oblige l'Algérie, dans un premier temps, à revenir au système de l'Association, dénoncé en 1971 : une dizaine d'accords ont été à ce jour 
conclus avec des sociétés étrangères dans le but de relancer les activités de recherche, de prospection et de forage. Cette timide ouverture n'ayant pas eu les effets escomptés, et la dette extérieure s'alourdissant, une nouvelle loi sur les hydrocarbures a été, on le sait, votée en novembre 1991, ouvrant plus largement les portes au capital étranger : celui-ci est désormais autorisé à participer jusqu'à hauteur de $49 \%$ à l'exploitation des gisements déjà en production.

Dans un autre domaine, le champ d'application des sociétés mixtes a été étendu, et c'est là une nouveauté remarquable en Algérie, au secteur du tourisme. Dans ce cadre, huit sociétés mixtes au moins ont été créées depuis l'année 1986, associant des capitaux privés algériens à des partenaires italiens, français, koweïtiens et même chinois.

Enfin, plus récemment, la promulgation en avril 1990 de la loi sur la Monnaie et le Crédit, dont on a rappelé plus haut les dispositions, vient modifier et compléter les lois précédentes votées en 1982 et en 1986, levant toutes restrictions à la pénétration du capital étranger, encouragé à investir librement et dans tous les secteurs d'activité : industries, banques et services, sans limitation de taux de participation. Des projets d'investissement ont été déposés dans ce cadre, certains ont même été agréés, mais semble-t-il, il n'y a pas encore de réalisation concrète. Il faudra sans doute attendre que l'Algérie sorte de ce climat d'incertitude et d'instabilité politiques qui prédomine en ce début d'année 1992 pour jauger l'impact de cette loi. Mais le mouvement de désengagement des capitaux étrangers observé dans les deux autres pays, pourtant réputés pour leur libéralisme, le Maroc et la Tunisie, et leur réorientation massive vers les pays d'Europe Centrale et de l'Est et des pays d'Asie, augure mal des chances qu'aurait l'Algérie d'attirer les investissements extérieurs.

Les risques d'éviction sont bien réels pour les trois pays du Maghreb.

\section{Investissements étrangers et type de spécialisation et d'insertion internationales}

42 Une approche désormais très répandue veut que toute tentative d'analyse macroéconomique des économies du tiers-monde en général, et de celles du Maghreb en particulier, passe par la trilogie désormais populaire: substitution aux importations, substitution aux exportations, promotion des exportations, à quoi on superpose parfois le type dit rentier.

43 Cette classification a un défaut rédhibitoire, elle n'est pas d'une grande fécondité analytique, car elle repose sur une vision purement phénoménologique de l'accumulation, et ne prend en compte qu'un aspect finalement peu significatif du régime d'accumulation : le type de stratégie et de politique industrielles.

Elle a pourtant un mérite: celui de mettre le doigt, et par conséquent d'attirer l'attention, sur l'une des caractéristiques essentielles de ces économies, à savoir leur mode de spécialisation et d'insertion internationales. Forme structurelle majeure au sens régulationniste du terme, le mode d'insertion internationale intervient d'une manière déterminante dans les configurations de l'accumulation interne, car c'est au niveau de cette instance que se matérialisent les contraintes externes qui s'exercent sur l'accumulation, en particulier les contraintes liées au financement extérieur.

Ce qui se dégage de la brève description empirique développée ci-dessus, c'est d'abord la relative insuffisance des flux d'investissements directs extérieurs vers le Maghreb, 
ensuite l'amorce de leur régression structurelle durant la dernière décennie, régression qui tendrait à s'aggraver durant les années 90, et enfin leur impact sectoriel peu dynamisant en termes d'accumulation.

Peut-on pour autant affirmer que les investissements étrangers provoquent des effets pervers à moyen/long terme ? C'est là une question à laquelle on ne saurait répondre sans un examen approfondi, et des formes structurelles qui conditionnent les modalités de régulation et des logiques d'accumulation dominantes, examen qui sortirait du cadre de cet article.

L'on se bornera, par conséquent, à avancer quelques hypothèses, en référence à la typologie évoquée plus haut.

1. Promotion des exportations, substitution aux importations, substitution aux exportations, économie rentière, ce schéma évoquant tout au plus des logiques d'accumulation, il y a lieu de se demander de prime abord si la localisation des investissements étrangers dans des activités d'extraction telle que le pétrole, des activités de service telles que le tourisme et les banques, ou dans des industries de main-d'oeuvre telles que le textile, n'est pas de nature à renforcer ces logiques d'accumulation basées sur l'extraversion, consolidant du coup ce type de spécialisation à faible valeur ajoutée propre aux économies sous-développées.

Il en résulterait alors un simple déplacement de contrainte, l'allégement provisoire de la contrainte de financement conduisant à l'alourdissement de la contrainte de réalisation, voire même de valorisation, puisque, dans cette logique, la dynamique d'accumulation est lourdement dépendante des aléas du marché extérieur, les activités de substitution d'importations pour leur approvisionnement en produits intermédiaires et en technologie, et la substitution d'exportations pour l'écoulement des produits primaires ou manufacturés.

Il faut insister ici sur la dépendance technologique, dont le modèle de substitution aux importations est porteur: elle est sans doute la plus redoutable de toutes, car elle hypothèque gravement l'avenir économique de ces pays en handicapant la constitution et le développement d'un potentiel scientifique et technologique local. Or la nouvelle carte des spécialisations internationales se joue d'abord au niveau de la maîtrise des nouvelles technologies.

51 Signalons enfin que la stratégie des firmes multinationales qui investissent dans des pays comme ceux du Maghreb, débouche souvent, lorsqu'elle n'est pas bien maitrisée par les États receveurs, sur un phénomène classique : implantation de «filiales-relais » et/ou de «filiales-ateliers », structures peu articulées aux économies locales et bases d'accumulation factices et instables.

52 2. Concernant les configurations du rapport salarial, autre forme structurelle de base qui, avec l'insertion internationale, conditionne les modalités internes de régulation et d'accumulation, deux hypothèses mériteraient d'être développées mais que nous ne ferons ici qu'évoquer très brièvement :

- Le type de rapport salarial mis en place dans le cadre des industries manufacturières comme le textile, intensives en main-d'oeuvre peu qualifiée à bas salaires, et cantonnée dans les tâches manuelles d'exécution, pourrait être qualifié du point de vue du procès du travail et de la division des tâches, de taylorisme primitif, au sens de Lipietz ${ }^{5}$. Ce type de rapport salarial, qui est basé sur la délocalisation partielle des seuls postes de travail parcellisés et répétitifs, et un appareillage peu coûteux, léger et individuel, s'accommode parfaitement 
d'une main-d'oeuvre facilement taylorisable (et facilement mobilisable) à partir du mode patriarcal de production domestique local: le salaire est considéré comme un revenu d'appoint et, par conséquent, se limite le plus souvent à sa partie directe.

- Le régime d'accumulation, ou du moins sa logique dominante, dont une telle modalité salariale est le support, s'apparenterait alors à ce qu'on pourrait appeler avec Lipietz, un fordisme périphérique $e^{6}$ ou plutôt un préformisme. Ce type d'accumulation est basé sur la recherche de gains de productivité élevés selon les normes du fordisme central, mais exclut une amélioration parallèle du pouvoir d'achat des salariés. Ceci permet une hausse de la rentabilité des capitaux investis dans ces activités - et nous avons vu plus haut (cf. p. 5) que cette rentabilité était meilleure au Maghreb que dans les autres zones d'investissement mais non un accroissement de la demande populaire et du marché interne.

53 Un tel modèle reste par conséquent cantonné dans d'étroites limites quant à la dynamique d'accumulation interne et ce, d'autant plus que la grande partie des profits tirés des investissements n'est par réinvestie sur place. On peut se demander, dans ces conditions, si ce système n'ajoute pas aux obstacles qui entravent la diffusion et l'épanouissement du rapport salarial pleinement capitaliste.

\section{Conclusion}

Une telle conclusion peut paraître négative, hors de saison, puisque aussi bien ce n'est pas d'une présence envahissante des investissements étrangers dont on saurait se plaindre au Maghreb, mais plutôt de leur absence.

5 D'ailleurs, le capital étranger n'est pas en soi porteur d'effets pervers, ni d'ailleurs d'effets bénéfiques. Tout dépend du contexte économique, social et même politique dans lequel il sera sollicité. A cet égard, on ne peut pas dire que la conjonction des processus en cours dans les pays du Maghreb soit favorable à une insertion bénéfique de l'investissement de capitaux extérieurs.

Tout est en effet déterminé par cette contrainte majeure qu'est la dette extérieure. Celle-ci - il serait inutile de rappeler ici son poids financier alarmant - est une des sources majeures de la crise, en même temps qu'elle prédétermine pour une grande part les mutations que connaissent ces pays.

7 Ce sont les exigences de la gestion de la dette qui imposent à l'ensemble des processus de recomposition en cours, au niveau des modalités de régulation et des formes d'accumulation, une logique imparable. Ramenées en effet à l'essentiel, les mécanismes d'ajustement structurel obéissent à une logique et une seule: permettre aux pays endettés d'assurer coûte que coûte la poursuite du service de la dette. La contrainte principale imposée ainsi à ces pays au travers de l'ajustement est d'abord de nature exogène, et c'est elle qui va surdéterminer les politiques de restructuration en profondeur des systèmes productifs. L'objectif poursuivi est de corriger les déséquilibres économiques et financiers, externes et internes, afin de parer au péril qui menace les pays endettés comme les pays créanciers : la cessation de paiement. Pour atteindre cet objectif, les politiques d'ajustement structurel, qu'elles soient dictées directement par le FMI (cas du Maroc et de la Tunisie) ou simplement inspirées des recommandations faites habituellement par cet organisme (cas de l'Algérie), visent d'un côté à comprimer la demande et, en priorité, la demande d'importations et, de l'autre côté, à stimuler l'offre d'exportations, source de devises. 

d'exportations (Algérie) ou par substitution d'exportations (Maroc et Tunisie), est inexorablement inscrite dans les réformes de structures en cours de réalisation et, en ce sens, la gestion de la dette extérieure tend à rapprocher les stratégies et à uniformiser les procédures d'ajustement des trois pays, au travers d'une spécialisation internationale perverse. Et, dans ces conditions, l'apport des investissements étrangers ne pourrait que conforter, voire aggraver, la tendance.

Certes, les conditions de refinancement (ou de "reprofilage ») de la dette extérieure devenant de plus en plus draconiennes, l'Algérie, le Maroc et la Tunisie, comme tous les autres pays débiteurs (qui n'ont pas eu la chance d'être du bon côté du manche lors de la guerre du Golfe) ne pourront pas se passer des investissements étrangers, qui eux, en principe, ne sont pas générateurs d'endettement ; mais ils auraient tort d'en attendre des miracles.

\section{NOTES}

1. Cf. sur ce sujet notre article «l'Union du Maghreb Arabe face à l'Europe des Douze : effets de domination et effet d'ajustement », Les Cahiers du CRISS, p. 33-58.

2. CHEVALLIER (A.) et KESSLER (V.), «Croissance et insertion internationale du Maghreb Questions sur l'avenir des relations avec l'Europe ", in Maghreb : les années de transition, op. cit. p. 265.

3. Cf. ABDOUN (R.), 1991, "Le capital étranger au Maghreb» in Les économies maghrébines et l'Europe, Actes des Rencontres de Grenoble, Les Cahiers du CRISS n 18, p. 127 sq.

4. KHROUZ (D.), 1990, Les investissements étrangers au Maroc, Ronéo.

5. LIPIETZ (A.), 1985, Mirages et miracles - Problèmes de l'industrialisation du Tiers-monde, Éd. La Découverte, Paris, p. 70.

6. LIPIETZ (A.), op. cit. p. 72.

\section{INDEX}

Mots-clés : Algérie, économie, Maghreb, Maroc, Tunisie

\section{AUTEUR}

LARBI TALHA

CNRSfr 